\title{
DNA methylation in ELOVL2 and C1orf132 correctly predicted chronological age of individuals from three disease groups
}

\author{
M. Spólnicka ${ }^{1}$ - E. Pośpiech ${ }^{2,3} \cdot$ B. Pepłońska ${ }^{4} \cdot$ R. Zbieć-Piekarska ${ }^{1} \cdot$ Ż. Makowska $^{1}$. \\ A. Pięta ${ }^{1}$ - J. Karlowska-Pik ${ }^{5}$ B. Ziemkiewicz ${ }^{5}$ - M. Wężyk ${ }^{4}$ - P. Gasperowicz ${ }^{6}$. \\ T. Bednarczuk ${ }^{7}$ - M. Barcikowska ${ }^{4} \cdot$ C. Żekanowski ${ }^{4} \cdot$ R. Ploski $^{6} \cdot$ Wojciech Branicki $^{1,3}$
}

Received: 19 December 2016 / Accepted: 4 July 2017 / Published online: 19 July 2017

(C) The Author(s) 2017. This article is an open access publication

\begin{abstract}
Improving accuracy of the available predictive DNA methods is important for their wider use in routine forensic work. Information on age in the process of identification of an unknown individual may provide important hints that can speed up the process of investigation. DNA methylation markers have been demonstrated to provide accurate age estimation in forensics, but there is growing evidence that DNA methylation can be modified by various factors including diseases. We analyzed DNA methylation profile in five markers from five different genes (ELOVL2, C1orf132, KLF14, FHL2, and TRIM59) used for forensic age prediction in three groups of individuals with diagnosed medical conditions. The obtained results showed that the selected agerelated $\mathrm{CpG}$ sites have unchanged age prediction capacity in the group of late onset Alzheimer's disease patients. Aberrant
\end{abstract}

Wojciech Branicki

wojciech.branicki@uj.edu.pl

1 Central Forensic Laboratory of the Police, Aleje Ujazdowskie 7, 00-583 Warsaw, Poland

2 Department of Genetics and Evolution, Institute of Zoology of the Jagiellonian University, Gronostajowa 9, 30-387 Krakow, Poland

3 Malopolska Centre of Biotechnology of the Jagiellonian University, Gronostajowa Gronostajowa st. 7A, 30-387 Krakow, Poland

4 Laboratory of Neurogenetics, Department of Neurodegenerative Disorders, Mossakowski Medical Research Center, Polish Academy of Sciences, 02-106 Warszawa, 5 Pawinskiego St, Warsaw, Poland

5 Faculty of Mathematics and Computer Science, Nicolaus Copernicus University, Chopina 12/18, 87-100 Toruń, Poland

6 Department of Medical Genetics, Centre for Biostructure, Medical University of Warsaw, Pawińskiego 3c, 02-106 Warsaw, Poland

7 Department of Internal Medicine and Endocrinology, Medical University of Warsaw, Banacha 1a, 02-097 Warsaw, Poland hypermethylation and decreased prediction accuracy were found for TRIM59 and KLF14 markers in the group of early onset Alzheimer's disease suggesting accelerated aging of patients. In the Graves' disease patients, altered DNA methylation profile and modified age prediction accuracy were noted for TRIM59 and FHL2 with aberrant hypermethylation observed for the former and aberrant hypomethylation for the latter. Our work emphasizes high utility of the ELOVL2 and C1orf132 markers for prediction of chronological age in forensics by showing unchanged prediction accuracy in individuals affected by three diseases. The study also demonstrates that artificial neural networks could be a convenient alternative for the forensic predictive DNA analyses.

Keywords DNA methylation · Chronological age · Alzheimer's disease · Graves' disease · Neural networks . Prediction accuracy

\section{Introduction}

Forensic intelligence through DNA analysis is now achievable when searching for an unknown individual in criminal, identification, or security cases. Wider use of the predictive DNA analysis methods in forensic investigations depends largely on their accuracy. Currently available predictive tests include biogeographic ancestry, pigmentation, hair loss and hair shape, extreme stature, facial morphology, and age [1]. All these methods are still being revised by using more advanced mathematical approaches, detailed studies of molecular mechanisms involved in phenotype determination and selection of additional predictors [2-6].

Age prediction has an important place in predictive DNA analysis. Informative itself, it can also increase prediction accuracy of several progressive physical appearance traits. 
Particularly, prediction of such appearance traits like hair graying, baldness, or skin wrinkles can be feasible only if biological age is included in prediction modeling.

Recent progress in epigenomics allowed identification of multiple DNA methylation loci that can be useful for age prediction [7-9]. Some of these markers have been used to develop age prediction models that may have a practical value in forensics [7, 10-13]. It has been shown that DNA methylation markers outperform the other types of potential age predictors including age-shortening telomeres, age-dependent changes in T cells' DNA, and age-altering mRNA level [14]. However, their use in routine forensic work still needs to be confirmed since prediction outcome can potentially be influenced by aberrations of DNA methylation patterns caused by various factors. It is worth noting that many of the identified DNA methylation markers used in age prediction are located in genes with known functions in Alzheimer's disease, cancer, tissue degradation, DNA damage, and oxidative stress [8]. There is growing evidence that factors like tobacco, alcohol, carcinogens, stress or infectious diseases, and even diet or physical activity can influence DNA methylation $[8,15]$. The potential age predictors may show different sensitivity to the influence of environment, medical history, or life style and thus show various capabilities to predict chronological age. Notably, Marioni et al. showed that faster aging predicted from DNA methylation status is heritable and predicts mortality independently of environmental or genetic factors [16]. Further research should explain whether particular DNA methylation markers might get misaligned as age predictors in individuals suffering from particular diseases or other external factors and thus be less suitable for accurate prediction of chronological age in diseased individuals. Indeed, it has been shown that DNA methylation status in some loci may depend on the number of cell divisions or can be influenced by other factors [17]. Thus, studies aiming to identify all potential players influencing differences in DNA methylation at particular loci between individuals at the same chronological age are important for better understanding the correlation between DNA methylation and age as well as for better accuracy of age prediction models. Exploration of this issue is important for age prediction reliability in routine forensic investigation.

In this study, we address the problem of age prediction accuracy of DNA methylation markers. We test DNA methylation status and prediction capacity of five literature age prediction markers studying three groups of individuals with different conditions including patients of early onset Alzheimer's disease (EOAD), late onset Alzheimer's disease (LOAD), and Graves' disease (GD). Our main motivation to study these groups was that all three diseases might potentially be associated with accelerated aging and affect age prediction accuracy parameters and thus perfectly fit with our aim to evaluate potential dysregulation of DNA methylation of markers used for chronological age prediction.

\section{Material and methods}

\section{Study samples and the protocol}

Written informed consent was obtained from AD and GD patients (or their legal representatives) and controls, according to the Declaration of Helsinki (BMJ 1991; 302:1194). The genetic study was approved by the Ethics Committee of the CSK-MSW Hospital (Warsaw, Poland) in compliance with national legislation and the Code of Ethical Principles for Medical Research Involving Human Subjects of the World Medical Association and at the Institute of Cardiology in Warsaw, no. IK-NP-0021-79/1396/13. Peripheral blood collected in EDTA-containing tubes was analyzed from 31 EOAD patients, 68 LOAD patients, and 91 GD patients. Detailed information about the tested groups is given in Table 1. Total DNA was extracted from the obtained blood samples using standard salting out procedure [18], the phenol/ chloroform method [19], or PrepFiler kit according to the manufacturer's directions (Applied Biosystems, Foster City, $\mathrm{CA})$. The total number of five $\mathrm{CpG}$ sites in five genes (ELOVL2, C1orf132, KLF14, TRIM59, and FHL2) were analyzed in the three groups using pyrosequencing technology. One to two micrograms of DNA was subjected to bisulfite conversion using the EpiTect 96 Bisulfite Kit according to the manufacturer's protocol (Qiagen, Hilden, Germany), and then, the previously applied PCR and sequencing protocols were used to measure DNA methylation status of the studied cytosines. In addition, DNAm data for 425 samples examined in our previous study [11] were used as a training set (305 samples) and healthy controls' testing set (120 samples).
Table 1 Characteristics of the testing set groups

\begin{tabular}{lclccc}
\hline Tested groups & Number & Mean Age \pm SD & Min age & Max age & Male (\%) \\
\hline Healthy controls [11] & 120 & $41.1 \pm 20.2$ & 2 & 75 & 47.5 \\
Late onset Alzheimer's disease (LOAD) & 68 & $70.9 \pm 3.4$ & 65 & 75 & 44.1 \\
Early onset Alzheimer's disease (EOAD) & 31 & $44.2 \pm 10.2$ & 31 & 68 & 48.4 \\
Graves' disease (GD) & 91 & $44.4 \pm 22.1$ & 12 & 76 & 34.1 \\
\hline
\end{tabular}




\section{Statistics}

DNA methylation profile in individuals from the tested disease groups

DNA methylation percentage measured for five age-related CpG sites (ELOVL2 c7, C1 orf132 c1, FHL2 c2, TRIM59 c7, KLF14 c1) was compared between individuals from three disease groups and matched healthy controls using independent sample Student's $t$ test. Because of the known differences in DNAm age correlation and age prediction accuracy between younger and older individuals [e.g., 11, 13], patients were divided into age group categories and calculations were performed for each age group separately. EOAD patients were divided into younger EOAD group (age 31-44) and older EOAD group (age 45-68); GD patients were categorized into younger GD group (age 12-30) and older GD group (56-76), while all LOAD patients accounted for only one age group category (age 65-75). Age categories are different for particular diseases due to different numbers of individuals at particular ages in various disease groups. Sizes of particular age group categories are provided in Table 2 . The healthy controls were selected from the available set of 120 controls taken from our previous study [11] and matched separately for the particular groups of EOAD, LOAD, and GD patients using criteria of mean age and age distribution between the compared groups. A proper distribution of age in the disease groups and healthy controls was confirmed with nonparametric Kolmogorov-Smirnov test. Analyses were performed using PS IMAGO 4 (IBM SPSS Statistics 24).

\section{Validation of predictive capacity of single DNAm markers in the tested disease groups}

In order to evaluate the predictive capacity of particular agerelated DNAm markers in the tested disease groups, separate prediction models for five selected CpGs were developed with 305 healthy individuals described in our previous study used as a training set [11]. In the present study, artificial neural network (ANN) approach was applied for prediction modeling instead of linear regression used before. ANN is a mathematical representation of the human neural architecture. It is composed of multiple nodes, called neurons, connected by links, which have assigned weights expressing the strength of the connections. Weights are adjusted in the process of neural network learning [50]. ANN models were developed in the form of multilayer perceptron (MLP) with one hidden layer and an automatically selected number of neurons (between 1 and 50). The activation functions were hyperbolic tangent for the hidden layer and identity for the output layer. For the remaining settings, default initial parameters of IBM SPSS Statistics were applied. The developed ANN prediction models for single DNAm markers were tested using groups of
EOAD, LOAD, and GD patients and age-matched healthy controls. Predicted age of individuals was compared with the true chronological age of individuals for the calculation of mean absolute error (MAE). Independent sample Student's $t$ test was used to compare mean predicted age and MAE calculated for the particular disease groups with age-matched healthy individuals. All the analyses were performed using PS IMAGO 4 (IBM SPSS Statistics 24).

\section{Multivariate ANN prediction model}

Finally, multivariate prediction model was developed based on all five selected age-related DNAm markers. Similarly to single $\mathrm{CpG}$ prediction models, 305 healthy individuals were used as a training set [11] and neural network approach was used for prediction modeling. Developed prediction model was tested using the same disease groups and age-matched healthy controls. Performance of the developed prediction model was evaluated throughout the calculation of MAE of predicted and chronological age and additionally percentage of correct predictions. Predictions were considered correct when the difference between actual and predicted age did not exceed \pm 5 years. This cutoff value was set according to the standard error of estimate calculated for the model to be at the level of 4.5 years [11]. Analyses were performed using PS IMAGO 4 (IBM SPSS Statistics 24).

\section{Results}

\section{DNA methylation status and predictive capacity of the single DNAm markers in the tested disease groups}

Three tested disease groups comprising of 31 early onset Alzheimer's disease patients, 68 late onset Alzheimer's disease patients, and 91 Graves' disease patients (Table 1) were used in this study to assess DNA methylation profile and predictive performance of five selected age-related $\mathrm{CpG}$ sites (ELOVL2 c7, C1orf132 c1, FHL2 c2, TRIM59 c7, KLF14 c1) in these disease groups. The results were compared to the age-matched healthy controls. All the selected markers were found to have unchanged DNA methylation status and age prediction capacity in the group of late onset Alzheimer's disease patients (Table 2).

Early onset Alzheimer's disease patients showed accelerated hypermethylation of TRIM59 $\mathrm{c} 7$ marker $(P=0.006)$, and the effect was most significant in younger EOAD group $(P=0.004)$. Aberrant hypermethylation of TRIM59 c7 led to decreased age prediction accuracy in EOAD patients, and again, the effect was most significant in younger EOAD group with $\mathrm{MAE}=12.2$ calculated for patients and $\mathrm{MAE}=5.7$ assigned for age-matched healthy controls $(P=0.008)$. When TRIM59 c7-based predicted age was compared, 
Table 2 DNA methylation status and age prediction accuracy of single age-related CpG sites measured in GD, EOAD, and GD patients compared to age-matched healthy controls

Locus CpG site Disease group Age group No. of patients No. of controls Mean \% of DNA methylation MAE of predicted and chronological

\begin{tabular}{|c|c|c|c|c|c|c|c|c|c|c|}
\hline & & & & & & & & age & & \\
\hline & & & & & Patients & Controls & $P$ value & Patients & Controls & $P$ value \\
\hline ELOVL2 c7 & GD & Younger & 44 & 39 & 60.34 & 57.59 & 0.055 & 3.918 & 3.861 & 0.935 \\
\hline & & Older & 47 & 33 & 83.43 & 82.76 & 0.529 & 6.390 & 7.960 & 0.299 \\
\hline & & Total & 91 & 72 & 72.26 & 69.13 & 0.136 & 5.195 & 5.740 & 0.522 \\
\hline & EOAD & Younger & 17 & 27 & 74.18 & 70.96 & 0.137 & 7.704 & 7.330 & 0.877 \\
\hline & & Older & 14 & 30 & 74.36 & 79.33 & 0.253 & 7.285 & 4.522 & 0.100 \\
\hline & & Total & 31 & 57 & 74.26 & 75.37 & 0.566 & 7.530 & 5.852 & 0.237 \\
\hline & LOAD & Total & 68 & 28 & 83.32 & 84.00 & 0.465 & 11.912 & 9.114 & 0.075 \\
\hline C1orf132 c1 & GD & Younger & 44 & 39 & 81.89 & 81.33 & 0.633 & 3.704 & 4.935 & 0.159 \\
\hline & & Older & 47 & 33 & 54.46 & 51.53 & 0.083 & 10.765 & 9.220 & 0.380 \\
\hline & & Total & 91 & 72 & 68.20 & 67.33 & 0.732 & 7.351 & 6.899 & 0.672 \\
\hline & EOAD & Younger & 17 & 27 & 68.94 & 68.26 & 0.772 & 7.833 & 6.498 & 0.472 \\
\hline & & Older & 14 & 30 & 59.00 & 56.20 & 0.279 & 7.384 & 6.640 & 0.652 \\
\hline & & Total & 31 & 57 & 64.45 & 61.91 & 0.236 & 7.631 & 6.573 & 0.387 \\
\hline & LOAD & Total & 68 & 28 & 48.44 & 50.61 & 0.281 & 11.326 & 11.273 & 0.967 \\
\hline FHL2 c2 & GD & Younger & 44 & 39 & 33.61 & 36.56 & 0.028 & 3.793 & 8.026 & 0.001 \\
\hline & & Older & 47 & 33 & 56.98 & 57.70 & 0.586 & 8.031 & 8.023 & 0.994 \\
\hline & & Total & 91 & 72 & 45.68 & 46.25 & 0.776 & 5.982 & 8.025 & 0.012 \\
\hline & EOAD & Younger & 17 & 27 & 41.59 & 41.30 & 0.900 & 8.836 & 8.012 & 0.709 \\
\hline & & Older & 14 & 30 & 48.57 & 51.93 & 0.119 & 9.047 & 5.539 & 0.215 \\
\hline & & Total & 31 & 57 & 44.74 & 46.89 & 0.256 & 8.931 & 6.711 & 0.198 \\
\hline & LOAD & Total & 68 & 28 & 60.60 & 60.43 & 0.889 & 10.243 & 8.357 & 0.060 \\
\hline TRIM59 c7 & GD & Younger & 44 & 39 & 30.43 & 26.24 & 0.0001 & 8.609 & 4.944 & 0.003 \\
\hline & & Older & 47 & 33 & 49.60 & 49.94 & 0.866 & 9.663 & 9.734 & 0.965 \\
\hline & & Total & 91 & 72 & 40.33 & 37.25 & 0.132 & 9.153 & 7.170 & 0.056 \\
\hline & EOAD & Younger & 17 & 27 & 40.76 & 35.00 & 0.004 & 12.176 & 5.653 & 0.008 \\
\hline & & Older & 14 & 30 & 48.93 & 44.23 & 0.013 & 6.805 & 7.264 & 0.803 \\
\hline & & Total & 31 & 57 & 44.45 & 39.86 & 0.006 & 9.751 & 6.500 & 0.023 \\
\hline & LOAD & Total & 68 & 28 & 54.32 & 52.86 & 0.385 & 9.970 & 10.033 & 0.955 \\
\hline KLF14 c1 & GD & Younger & 44 & 39 & 4.20 & 4.56 & 0.261 & 5.082 & 8.873 & 0.002 \\
\hline & & Older & 47 & 33 & 10.11 & 11.18 & 0.083 & 12.101 & 10.751 & 0.414 \\
\hline & & Total & 91 & 72 & 7.25 & 7.60 & 0.564 & 8.707 & 9.734 & 0.344 \\
\hline & EOAD & Younger & 17 & 27 & 10.18 & 6.74 & 0.013 & 14.478 & 9.076 & 0.096 \\
\hline & & Older & 14 & 30 & 8.07 & 9.43 & 0.206 & 14.366 & 9.714 & 0.035 \\
\hline & & Total & 31 & 57 & 9.23 & 8.16 & 0.239 & 14.427 & 9.415 & 0.016 \\
\hline & LOAD & Total & 68 & 28 & 13.24 & 11.89 & 0.102 & 13.247 & 12.234 & 0.653 \\
\hline
\end{tabular}

Significant values marked with italics

patients in younger EOAD group were found to have significantly increased age (9 years on average) when comparing to age-matched healthy controls $(P=0.004$, Fig. 1$)$. Increased methylation was also found in younger EOAD group for KLF14 c1 ( $P=0.013$, Table 2). Although no prediction accuracy deviation expressed by MAE was observed $(P=0.096)$ for patients included in younger EOAD group, they were found to have significantly increased age (by 10.1 years) when predicted age of patients and controls was directly compared $(P=0.012$, Fig. 1). Marginal deviation in MAE was observed for older EOAD group $(P=0.035)$, but difference was insignificant when predicted age was compared $(P=0.093)$.

Aberrant DNA methylation pattern was also observed in the group of GD patients. Similarly to EOAD patients, accelerated hypermethylation was observed for TRIM59 c7. The effect was significant in younger GD group $(P=0.0001$, 
Fig. 1 Predicted age in younger EOAD group and age-matched healthy controls. Prediction analysis was performed with KLF14 $\mathrm{c} 1$ and TRIM59 $\mathrm{c} 7$ markers

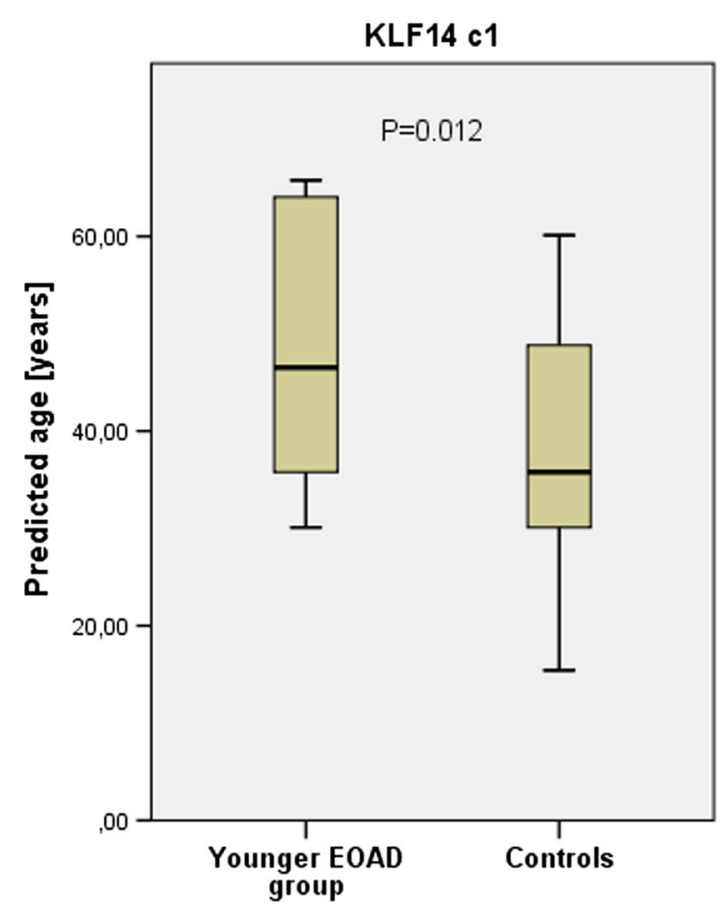

KLF14 c1
TRIM59 c7

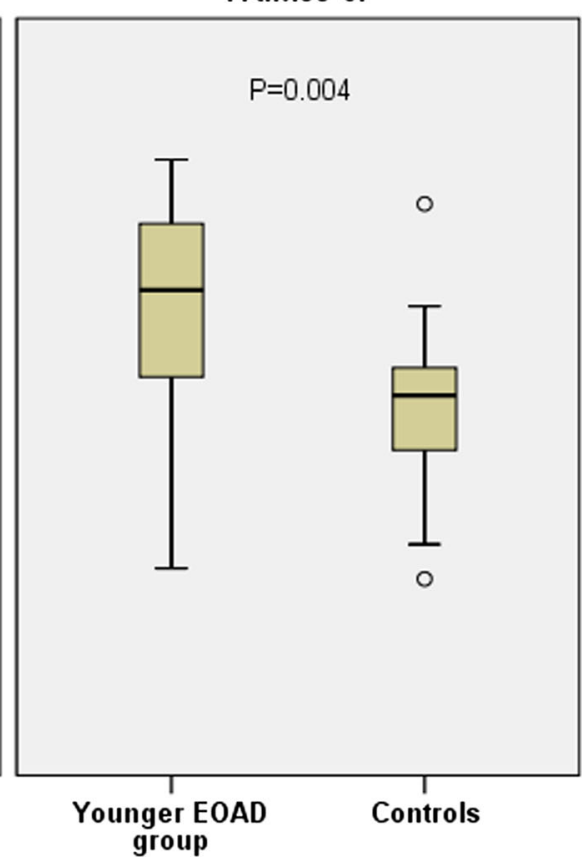

Table 2). Prediction analysis performed using TRIM59 c7 marker showed significantly decreased age prediction accuracy in younger GD group (MAE $=8.6$ ) when comparing to healthy controls $(\mathrm{MAE}=4.9, P=0.003$ ) with younger GD patients predicted to be 6 years older than controls $(P=0.0001$, Fig. 2$)$. In contrast, decreased methylation was noticed for younger GD group when FHL2 c2 marker was analyzed $(P=0.028)$. Significant difference in MAE was observed between younger GD group $(\mathrm{MAE}=3.8)$ and controls (MAE $=8, P=0.001)$, and patients in this group were predicted 4.9 years younger than controls $(P=0.008$, Fig. 2$)$. Ambiguous result was obtained for KLF14 c1 marker. Although significantly decreased age prediction accuracy measured by MAE was observed in younger GD group comparing to control group $(P=0.002)$, no significant difference was observed in DNA methylation status $(P=0.261)$ and no significant difference in mean predicted age was noted ( $P=0.224$, Fig. 2$)$.

Unchanged DNA methylation status and predictive performance were detected for the two remaining markers, ELOVL2 $\mathrm{c} 7$ and C1orf132 $\mathrm{c} 1$ in the three disease groups and all age group categories.

\section{Prediction modeling using five DNAm loci}

In the next step, multivariate prediction model was developed including all five age-related CpGs. Developed multivariate ANN model predicted age of total EOAD patients with significantly decreased accuracy (MAE $=7.1$ ) when comparing to the healthy control group (MAE $=3.8, P=0.002$, Table 3 ). When different age categories were analyzed, this effect was observed only in younger EOAD group $(P=0.011)$. The number of correct predictions was also decreased in the total EOAD group (38.7\%) when comparing to the healthy controls (70.2\%), and again, the effect was noticeable particularly in younger EOAD group (Table 3). On average, patients in the total EOAD group were predicted to be 1.7 years older than the chronological age of patients but 6.4 years older than the chronological age of patients when only younger EOAD group was taken into account (Fig. 3a). In contrast, healthy controls were predicted to be on average 0.38 years younger than the true chronological age of individuals, and prediction accuracy did not dropped when only younger individuals (below 45 years old) were taken into account (predicted 0.12 years younger than the true chronological age, Fig. 3a). When predicted age was compared between patients and controls, patients included in younger EOAD group were predicted to be at significantly higher age (by 5.8 years) than matched controls $(P=0.013$, Fig. $3 b)$.

No deviation in MAE and mean predicted age was noted in the remaining study groups, that is LOAD and GD patients when multivariate NN prediction model was applied (Table 3).

\section{Discussion}

Difference between chronological and biological age of an individual is important in medical and forensic studies. Biological age is relevant for onset and progression rate of many diseases. It has been suggested that delaying the rate of biological aging may have a positive overall impact on 
Fig. 2 Predicted age in younger GD group and age-matched healthy controls. Prediction analysis was performed with FHL2, KLF14 c1, and TRIM59 c7 markers

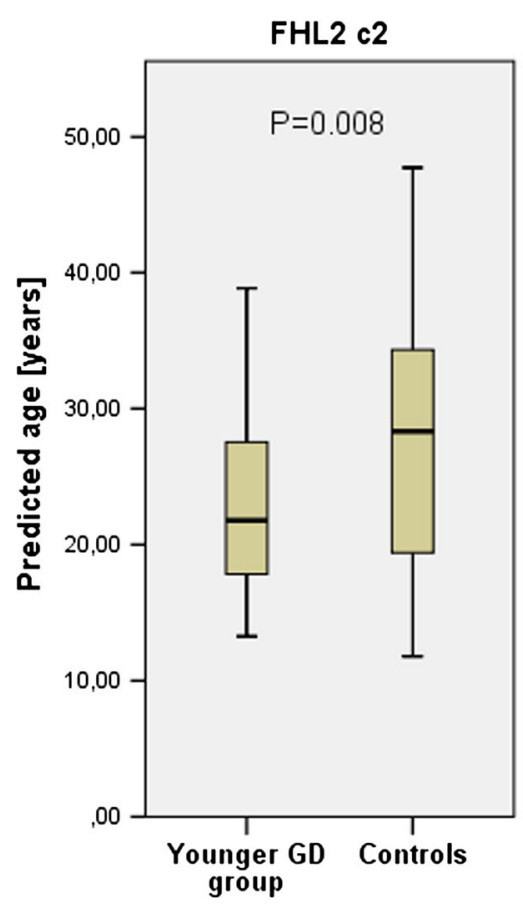

KLF14 c1

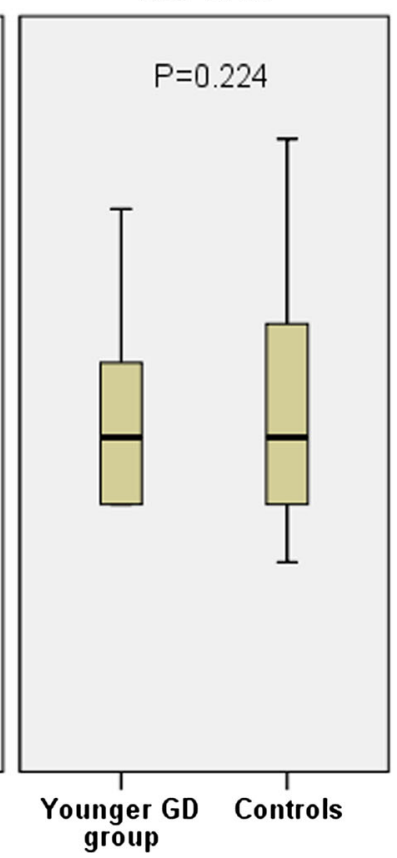

TRIM59 c7

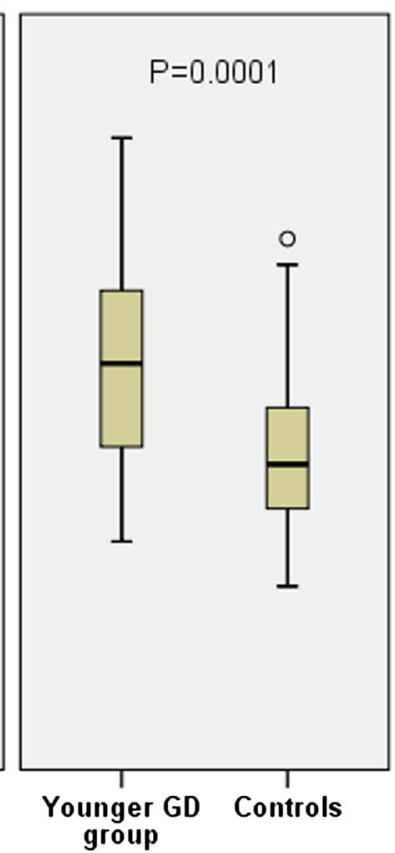

the life expectancy and quality [20, 21]. In forensics, prediction of chronological and biological age is important. Besides direct use of chronological age for intelligence purposes, information about biological age can strengthen prediction of progressive appearance traits, which due to a recent progress in genetics can be predicted from DNA left at the crime scene. Differentiation between DNA methylation markers, which depend solely on the number of cell replications and those modified by other factors, may be important for developing more accurate models predicting chronological age in forensics. Evaluation of age markers may be accomplished by studying their response to various environmental factors and diseases that may change DNA methylation affecting accuracy of prediction of calendar age. Such markers should be avoided in prediction models aiming at prediction of chronological age.

\section{Validation of age predictive markers}

In this research study, three groups of patients, EOAD, LOAD, and GD, were investigated using pyrosequencing technology and ANN prediction model based on five previously selected age-correlated CpG sites in ELOVL2, C1orf132, KLF14,
Table 3 MAE and percentage of correct predictions in EOAD, LOAD, and GD patients compared to age-matched healthy controls

\begin{tabular}{|c|c|c|c|c|c|}
\hline Disease group & Age category & Parameter & Patients & Controls & $P$ value \\
\hline \multirow[t]{6}{*}{ GD } & \multirow[t]{2}{*}{ Younger } & MAE & 2.5 & 2.7 & \multirow[t]{2}{*}{0.595} \\
\hline & & Correct predictions $(\%)$ & $93.2(41 / 44)$ & $87.2(34 / 39)$ & \\
\hline & \multirow[t]{2}{*}{ Older } & MAE & 6.1 & 4.4 & \multirow[t]{2}{*}{0.106} \\
\hline & & Correct predictions $(\%)$ & $55.3(26 / 47)$ & $60.6(20 / 33)$ & \\
\hline & \multirow[t]{2}{*}{ Total } & MAE & 4.4 & 3.5 & \multirow[t]{2}{*}{0.146} \\
\hline & & Correct predictions $(\%)$ & $73.6(67 / 91)$ & $75.0(54 / 72)$ & \\
\hline \multirow[t]{6}{*}{ EOAD } & Younger & MAE & 7.3 & & \multirow[t]{2}{*}{0.011} \\
\hline & & Correct predictions $(\%)$ & $29.4(5 / 17)$ & $66.7(18 / 27)$ & \\
\hline & Older & MAE & 7.0 & 3.4 & \multirow[t]{2}{*}{0.066} \\
\hline & & Correct predictions (\%) & $50.0(7 / 14)$ & $73.3(22 / 30)$ & \\
\hline & Total & MAE & 7.1 & 3.8 & \multirow[t]{2}{*}{0.002} \\
\hline & & Correct predictions (\%) & $38.7(12 / 31)$ & $70.2(40 / 57)$ & \\
\hline \multirow[t]{2}{*}{ LOAD } & Total & MAE & 6.1 & 5.6 & \multirow[t]{2}{*}{0.382} \\
\hline & & Correct predictions (\%) & $76.5(52 / 68)$ & $50.0(14 / 28)$ & \\
\hline
\end{tabular}

Prediction analysis performed with multivariate ANN model. Significant values marked with italics 
A

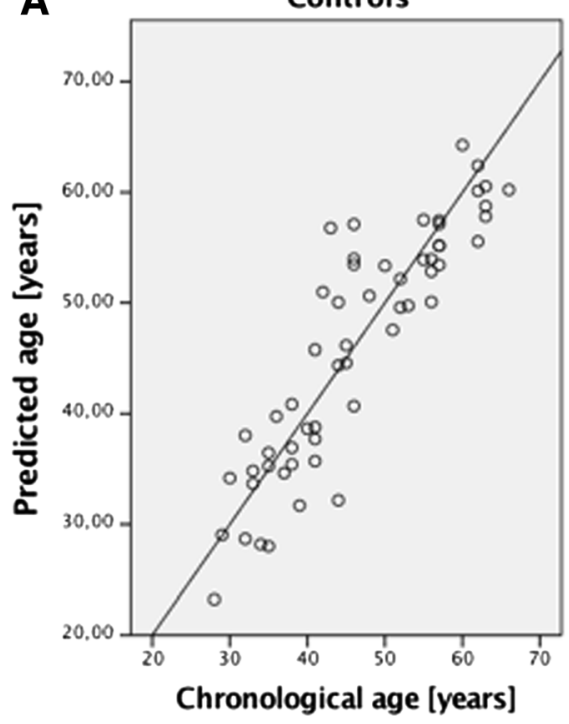

Fig. 3 Age prediction analysis in EOAD patients using multivariate ANN model. a Predicted vs chronological age in total EOAD group
EOAD

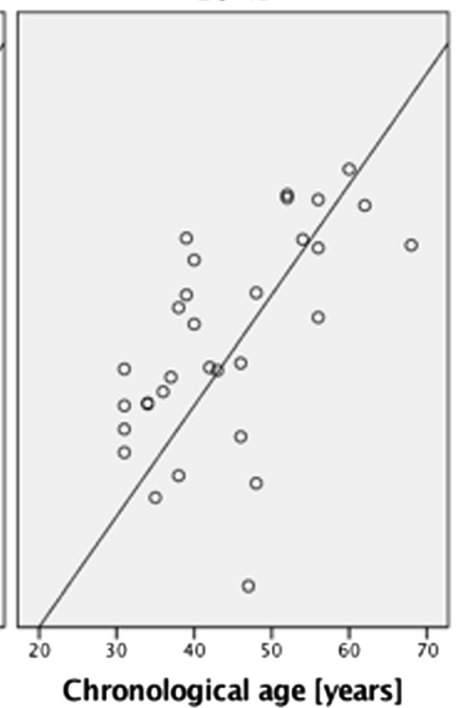

B

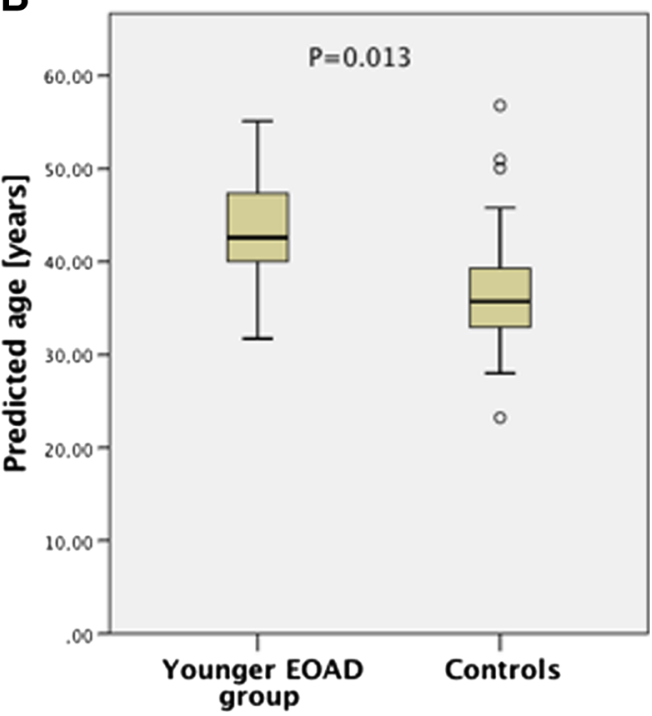

and age-matched healthy controls. b Predicted age comparison between younger EOAD group and age-matched healthy controls

FHL2, and TRIM59. Significant differences of age prediction capacity were noted for particular markers in the case of EOAD and GD patients, and they influenced the overall age prediction in EOAD group. The effects were more obvious in the younger groups of investigated patients, and these may be caused by the previously described general deregulation of age-related DNA methylation markers in elderly individuals $[11,13]$.

The overall accuracy of multivariate ANN age prediction model was significantly decreased in EOAD patients as measured by MAE and the number of positive predictions. The mean predicted age of patients was higher than the true chronological age of patients and higher than the mean age of agematched healthy controls. The remaining study groups of LOAD and GD patients were predicted with accuracy observed in the age-matched healthy controls when multivariate ANN age prediction model was applied.

In order to get a better insight into age prediction accuracy of the model, separate examination of prediction accuracy of single five age predictors was undertaken in the three investigated groups of individuals. The analysis disclosed significant loss of accuracy as measured by MAE in the case of TRIM59, KLF14, and FHL2. The changed prediction capacity was noted not only in the group of EOAD but also in the younger individuals suffering from GD. TRIM59 and KLF14 were found to be hypermethylated in EOAD patients indicating older age of the investigated individuals. Thus, the worse performance of the overall prediction model was caused by deregulation of DNA methylation in these two loci. Interestingly, TRIM59 was also hypermethylated in patients included in the younger GD group, but this effect was not observed when investigating the overall performance of the model, because of the balancing influence of hypomethylation in the FHL2 locus.
Alzheimer's disease is the most common type of dementia characterized by massive neuronal loss caused by overproduction of $\beta$-amyloid and hyperphosphorylated microtubuleassociated protein tau accumulated into senile plaques and neurofibrillary tangles, respectively. Senile plaques with minimal cortical tau pathology and no accompanying history of cognitive decline are also hallmarks of pathological aging. Moreover, $\beta$-amyloid accumulation in both conditions is remarkably similar [22]. There are two main types of AD: EOAD and LOAD. Familial EOAD represents $1-5 \%$ of all cases of $\mathrm{AD}$ and in $40 \%$ is associated with mutations in the genes PSEN1, PSEN2, and APP. The group of EOAD patients studied here has been well characterized genetically [23-25]. The genetics of LOAD is still poorly understood, but significant progress has been made by the GWAS analyses, which have identified 25 genes to be associated with this type of $\mathrm{AD}$ [26]. The involvement of DNA methylation in AD is still under debate, especially its causal or subsequent role remains unclear [27]. However, growing evidence shows $\mathrm{AD}$ as being associated with DNA hypermethylation and histone deacetylation, suggesting a general repressed chromatin state and epigenetically reduced plasticity in AD [28]. Generally, analysis of DNA methylation in AD patients revealed differences in brain tissue, but results for peripheral blood are conflicting $[29,30]$. In a medical sense, our result on methylation differences in aging markers further emphasizes differences between the two groups of Alzheimer's disease patients.

GD is an autoimmune disorder, which affects up to $2 \%$ of the European population. Antibody-driven activation of the thyrotropin receptor leads to hyperfunction of the thyroid gland and thyroid enlargement and in consequence increased production of the thyroid hormone [31]. Previous study 
investigated $\mathrm{T}$ cell receptor rearrangement excision circle (sjTREC) concentration in Graves' disease patients and reported that although generally decreasing with age, it was significantly higher in GD group compared with controls [32]. Examination of the decrease in the number of sjTREC molecules caused by thymus involution occurring in the course of human life has a predictive capacity and has been implemented in forensic age prediction [33]. Our investigation of age predictive DNA methylation markers does not support a conclusion that can be drawn from the study performed by Strawa about younger age of GD patients [32]. A hypomethylation of the FHL2 gene predicts younger age of patients, but two other markers (TRIM59 and KLF14) have increased methylation comparing to the controls, while ELOVL2 and C1orf132 are unchanged.

The FHL2 encodes a multifunctional adaptor protein that is involved in the regulation of gene transcription, signal transduction, and cell proliferation and differentiation [34]. Interestingly, FHL2 may act depending on a tissue as an oncoprotein or as a tumor suppressor [35]. It has been reported that FHL2 is involved in colorectal, gastric, and pancreatic cancer and hepatocellular carcinoma [36, 37]. The FHL2 protein was found to interact with presenilin 1 and presenilin 2, both involved in $\mathrm{AD}[38,39]$.

The TRIM59 gene was hypermethylated in both EOAD and GD patients. This gene is involved in cancer that has been suggested as multitumor marker detecting early tumorigenesis [40]. TRIM59, which encodes an ubiquitin ligase, might be involved in neurodegeneration process by affected proteostasis, for instance by contribution to accumulation of neurofilament light chain, similarly to TRIM2 [41]. Several data suggested pro-apoptotic cooperation of p53 and TRIM59, and activation of p53 signaling is commonly known to leading to death of post-mitotic neurons in Alzheimer's disease patients. A physical interaction of TRIM59 and p53 under TRIM59 upregulation resulted with ubiquitination and degradation of p53 [42].

The methylation marker KLF14 was found to have decreased age prediction accuracy in EOAD and GD patients. However, the results were ambiguous as no significant change in DNA methylation was observed in particular age group categories, and therefore, this effect needs to be further validated on a larger sample size. Loss of KLF14 in mouse was shown to cause centrosome amplification and tumorigenesis, and it has been suggested that reduction of KLF14 may increase the risk of breast cancer and colon cancer [43]. KLF family was found to be involved in transcriptional modulation of neuronal genes, e.g., dopamine D2 receptor [44]. Importantly, KLF14 reduction was reported to be responsible for aneuploidy and finally tumorigenesis [43]. It is very likely that this could be linked with increased DNA damage stress response, commonly observed in $\mathrm{AD}$, that could be further linked with p53-mediated post-mitotic apoptosis, as also proposed by [45].
It is worth noting that age-associated changes in DNA methylation patterns may have regulatory role on gene activity and developmental processes. Recently, it has been shown that the mean age-associated DNA methylation patterns manifests in early childhood (2-16 years) and the majority of ageassociated loci involved increased DNA methylation resulting in decreased gene expression [46]. Authors concluded that the results could pinpoint genes susceptible to aging-related disease-associated epigenetic dysregulation.

Markers epigenetically regulated in diseases should be avoided in prediction models developed for estimation of chronological age. However, it seems that each DNA methylation age predictor may have a unique sensitivity to various external factors. Our recent study involving the same prediction model discovered significantly lower predicted age of patients after hematopoietic stem cell transplantation (HSCT) [47]. This result was discordant to the report of Weidner et al., and detailed analysis of our results showed that the observed discrepancy is caused by different sets of predictors used in both studies [48]. In particular, the effect observed in our study was a result of hypermethylation of a single $\mathrm{C} 1$ orf 132 locus, while the remaining loci included in the model did not show this pattern. It means that in some cases, like HSCT, C1orf132, which encodes for long noncoding RNA MIR29B2C, may not be a good predictor of chronological age.

However, in the present research study, C1orf132 and ELOVL2 were found to be stable age markers in three groups of patients. It is worth noting that HSCT is a very rare medical treatment $(\sim 50,000$ worldwide HSCTs in 2006$)$, while the incidence of GD is 20 to 50 cases per year, per 100,000 individuals $[49,50]$. ELOVL2 is the most thoroughly validated age marker $[4,8,11,51]$. Horvath demonstrated that cell passaging in general increases DNA methylation age, but he also rejected the hypothesis that DNA methylation age is the same as mitotic age (the number of cell divisions) as it tracks chronological age in cell types which not proliferate. Therefore, Horvath proposed that DNA methylation reflects rather the work done by an organism to maintain epigenetic stability [52]. On the other hand, Bacalini et al. have reported differences in age-associated methylation change schemes in two known age predictors: ELOVL2 and FHL2. The study showed that ELOVL2 methylation is a marker of cell divisions occurring during human aging and not the senescence [17]. Contrary, DNA methylation of the FHL2 gene was not strictly associated to cell divisions in that research. This means that DNA methylation in different loci may indeed depend on various factors, and there is a chance that some predictors may better measure chronological age (mitotic age) than the others. The study of Bacallini shows that methylation of FHL2 can be affected by other age-associated factors, that is also supported by our study of GD patients. Our study may help in selection of the most optimal marker set for chronological age prediction in forensics, but due to a small number of samples in 
particular disease age groups, the findings need to be replicated. Overall, the study reinforces the significance of ELOVL2 and $\mathrm{C} 1$ orf 132 as predictors of chronological age in forensic investigations. Further studies exploring other potential confounders of DNA methylation will be particularly interesting in the case of C1orf132 that is a very powerful age predictor. This locus encodes for a long noncoding RNA and may play a role in epigenetic regulation. As mentioned earlier, our previous study demonstrated its potential involvement in graft function after HSCT [47].

\section{Prediction modeling using artificial neural network approach}

The artificial neural networks used in the study for prediction modeling seem to be a good alternative for the traditional parametric methods like linear regression. ANN can be a simple solution to eliminate a problem of nonlinear patterns that can be attributed to particular DNAm markers [53]. Many authors highlight different advantages of neural networks: the ability to recognize and learn all types of relations, lack of assumptions on the distribution of the input data, automatic handling of variable interactions, or the high tolerance to missing and noisy data [54-57]. We demonstrated its superiority over regression methods in our previous reports on hair morphology [2] and eye color prediction [3]. Recently, ANN has also been shown to be more accurate in age prediction modeling $[58,59]$. For comparison purposes, calculations in the present study were performed with linear regression confirming the general outcome of the study but demonstrating slightly lower prediction accuracy parameters when comparing to the neural networks approach (data not present).

\section{Forensic impact of the study}

The multivariate ANN age prediction model failed to predict age accurately in case of EOAD patients. Since EOAD is a very rare condition that affects merely $1-5 \%$ of $A D$ patients, this finding has a minor impact on forensic age prediction. However, separate analyses of prediction capacity of five markers included in the evaluated model clearly showed that three markers-TRIM59, KLF14, and FHL2 - revealed altered DNA methylation patterns in EOAD and GD groups. Although this finding does not disqualify the three markers as predictors of chronological age, further studies shall investigate their sensitivity to various factors affecting biological age of an individual. Importantly, our study showed that the two important age predictors-ELOVL2 and C1orf132-keep their high predictive capacity in three groups of patients. Our finding confirms conclusion from a recent study reporting DNA methylation in ELOVL2 to depend solely on the number of cell divisions and FHL2 to depend on other age-related factors [17]. This study shows that C1orf132, a very powerful age predictor, may together with ELOVL2 comprise a good foundation for prediction of chronological age in forensics, although further studies are necessary especially for C1orf132 since in our previous investigation, this locus showed changed DNA methylation in the group of individuals after HSCT.

Acknowledgements The study was supported by a grant from the National Centre for Research and Development in Poland, no. DOBR/0002/R/ID1/2012/03. Authors wish to thank participants of the study for providing samples.

Open Access This article is distributed under the terms of the Creative Commons Attribution 4.0 International License (http:// creativecommons.org/licenses/by/4.0/), which permits unrestricted use, distribution, and reproduction in any medium, provided you give appropriate credit to the original author(s) and the source, provide a link to the Creative Commons license, and indicate if changes were made.

\section{References}

1. Kayser M (2015) Forensic DNA phenotyping: predicting human appearance from crime scene material for investigative purposes. Forensic Sci Int Genet 18:33-48

2. Pośpiech E, Karłowska-Pik J, Marcińska M et al (2015) Evaluation of the predictive capacity of DNA variants associated with straight hair in Europeans. Forensic Sci Int Genet 19:280-288. doi:10.1016/ j.fsigen.2015.09.004

3. Pośpiech E, Karłowska-Pik J, Ziemkiewicz B et al (2016) Further evidence for population specific differences in the effect of DNA markers and gender on eye colour prediction in forensics. Int $\mathrm{J}$ Legal Med 130:923-934. doi:10.1007/s00414-016-1388-2

4. Bekaert B, Kamalandua A, Zapico SC et al (2015) Improved age determination of blood and teeth samples using a selected set of DNA methylation markers. Epigenetics 10:922-930

5. Visser M, Kayser M, Palstra RJ (2012) HERC2 rs12913832 modulates human pigmentation by attenuating chromatin-loop formation between a long-range enhancer and the OCA2 promoter. Genome Res 22:446-455

6. Santos C, Phillips C, Fondevila M et al (2016) Pacifiplex: an ancestry-informative SNP panel centred on Australia and the Pacific region. Forensic Sci Int Genet 20:71-80

7. Bocklandt S, Lin W, Sehl ME et al (2011) Epigenetic predictor of age. PLoS One 6:e14821

8. Hannum G, Guinney J, Zhao L et al (2013) Genome-wide methylation profiles reveal quantitative views of human aging rates. Mol Cell 49:359-376

9. Johansson A, Enroth S, Gyllensten U (2013) Continuous aging of the human DNA methylome throughout the human lifespan. PLoS One 8:e67378

10. Weidner CI, Lin Q, Koch CM et al (2014) Aging of blood can be tracked by DNA methylation changes at just three $\mathrm{CpG}$ sites. Genome Biol 15:R24

11. Zbieć-Piekarska R, Spólnicka M, Kupiec T et al (2015) Development of a forensically useful age prediction method based on DNA methylation analysis. Forensic Sci Int Genet 17:173-179. doi:10.1016/j.fsigen.2015.05.001

12. Lee HY, Jung SE, Oh YN et al (2015) Epigenetic age signatures in the forensically relevant body fluid of semen: a preliminary study. Forensic Sci Int Genet 19:28-34. doi:10.1016/j.fsigen.2015.05.014

13. Freire-Aradas A, Phillips C, Mosquera-Miguel A et al (2016) Development of a methylation marker set for forensic age 
estimation using analysis of public methylation data and the Agena Bioscience EpiTYPER system. Forensic Sci Int Genet 24:65-74. doi:10.1016/j.fsigen.2016.06.005

14. Zubakov D, Liu F, Kokmeijer I et al (2016) Human age estimation from blood using mRNA, DNA methylation, DNA rearrangement, and telomere length. Forensic Sci Int Genet 24:33-43. doi:10.1016/ j.fsigen.2016.05.014

15. Mathers JC, Strathdee G, Relton CL (2010) Induction of epigenetic alterations by dietary and other environmental factors. Adv Genet 71:3-39. doi:10.1016/B978-0-12-380864-6.00001-8

16. Marioni RE, Shah S, McRae AF et al (2015) The epigenetic clock is correlated with physical and cognitive fitness in the Lothian Birth Cohort 1936. Int J Epidemiol 44:1388-1396

17. Bacalini MG, Deelen J, Pirazzini C et al (2016) Systemic ageassociated DNA hypermethylation of ELOVL2 gene: in vivo and in vitro evidences of a cell replication process. J Gerontol A Biol Sci Med Sci. doi:10.1093/gerona/glw185

18. Miller SA et al (1988) A simple salting out procedure for extracting DNA from human nucleated cells. Nucleic Acids Res 16:1215

19. Köchl S, Niederstätter H, Parson W (2005) DNA extraction and quantitation of forensic samples using the phenolchloroform method and real-time PCR. Methods Mol Biol 297:13-30

20. Kaeberlein M, Rabinovitch PS, Martin GM (2015) Healthy aging: the ultimate preventative medicine. Science 350:1191-1193

21. Niccoli T, Partridge L (2012) Ageing as a risk factor for disease. Curr Biol 22:R741-R752

22. Moore BD, Chakrabarty P, Levites Y et al (2012) Overlapping profiles of $A \beta$ peptides in the Alzheimer's disease and pathological aging brains. Alzheimers Res Ther 4:18

23. Zekanowski C, Styczyńska M, Pepłońska B et al (2003) Mutations in presenilin 1, presenilin 2 and amyloid precursor protein genes in patients with early-onset Alzheimer's disease in Poland. Exp Neurol 184:991-996

24. Zekanowski C, Religa D, Safranow K et al (2005) The -22c/t polymorphism in presenilin 1 gene is not connected with late-onset and early-onset familial Alzheimer's disease in Poland. J Neural Transm 112:839-845

25. Bialopiotrowicz E, Kuzniewska B, Kachamakova-Trojanowska N et al (2011) Cell cycle regulation distinguishes lymphocytes from sporadic and familial Alzheimer's disease patients. Neurobiol Aging 32:2319.e13-2319.e26. doi:10.1016/j.neurobiolaging.2010. 04.017

26. Cuyvers E, Sleegers K (2016) Genetic variations underlying Alzheimer's disease: evidence from genome-wide association studies and beyond. Lancet Neurol 15:857-868

27. Sanchez-Mut JV, Heyn H, Vidal E et al (2016) Human DNA methylomes of neurodegenerative diseases show common epigenomic patterns. Transl Psychiatry 6:e718

28. Sanchez-Mut JV, Gräff J (2015) Epigenetic alterations in Alzheimer's disease. Front Behav Neurosci 9:347

29. Tannorella P, Stoccoro A, Tognoni G et al (2015) Methylation analysis of multiple genes in blood DNA of Alzheimer's disease and healthy individuals. Neurosci Lett 600:143-147

30. Di Francesco A, Arosio B, Falconi A et al (2015) Global changes in DNA methylation in Alzheimer's disease peripheral blood mononuclear cells. Brain Behav Immun 45:139-144

31. Płoski R, Szymański K, Bednarczuk T (2011) The genetic basis of Graves' disease. Curr Genomics 12:542-563

32. Strawa K, Markowska A, Miśkiewicz P et al (2014) Increased concentration of T-cell receptor rearrangement excision circles (TREC) in peripheral blood in Graves' disease. Clin Endocrinol 81:769-774

33. Zubakov D, Liu F, VanZelm MC et al (2010) Estimating human age from T-cell DNA rearrangements. Curr Biol 20:R970-R971
34. Kadrmas JL, Beckerle MC (2004) The LIM domain: from the cytoskeleton to the nucleus. Nat Rev Mol Cell Biol 5: 920-931

35. Verset L, Feys L, Trépant AL (2016) FHL2: a scaffold protein of carcinogenesis, tumour-stroma interactions and treatment response. Histol Histopathol 31:469-478

36. WangJ YY, Xia HH et al (2007) Suppression of FHL2 expression induces cell differentiation and inhibits gastric and colon carcinogenesis. Gastroenterology 132:1066-1076

37. Amann T, Egle Y, Bosserhoff AK et al (2010) FHL2 suppresses growth and differentiation of the colon cancer cell line HT-29. Oncol Rep 23:1669-1674

38. McCarthy (2005) Involvement of presenilins in cell-survival signalling pathways. Biochem Soc Trans33:568-572

39. Tanahashi H, Tabira T et al (2000) Alzheimer's disease-associated presenilin 2 interacts with DRAL, an LIM-domain protein. Hum Mol Genet 9:2281-2289

40. Khatamianfar V, Valiyeva F, Rennie PS et al (2012) TRIM59, a novel multiple cancer biomarker for immunohistochemical detection of tumorigenesis. BMJ Open 2:e001410. doi:10.1136/ bmjopen-2012-001410

41. Balastik M, Ferraguti F, Pires-da Silva A et al (2008) Deficiency in ubiquitin ligase TRIM2 causes accumulation of neurofilament light chain and neurodegeneration. Proc Natl Acad Sci U S A 105: 12016-12021

42. Zhou Z, Ji Z, Wang Y et al (2014) TRIM59 is up-regulated in gastric tumors, promoting ubiquitination and degradation of $\mathrm{p} 53$. Gastroenterology 147:1043-1054. doi:10.1053/j.gastro.2014.07. 021

43. Fan G, Sun L, Shan P et al (2015) Loss of KLF14 triggers centrosome amplification and tumorigenesis. Nat Commun 6:8450

44. Seo S, Lomberk G, Mathison A et al (2012) Krüppel-like factor 11 differentially couples to histone acetyltransferase and histone methyltransferase chromatin remodeling pathways to transcriptionally regulate dopamine D2 receptor in neuronal cells. J Biol Chem 287:12723-12735. doi:10.1074/jbc.M112.351395

45. Ohashi A, Ohori M, Iwai K et al (2015) Aneuploidy generates proteotoxic stress and DNA damage concurrently with p53mediated post-mitotic apoptosis in SAC-impaired cells. Nat Commun 6:7668. doi:10.1038/ncomms8668

46. Gervin K, Andreassen BK, Hjorthaug HS et al (2016) Intraindividual changes in DNA methylation not mediated by cell-type composition are correlated with aging during childhood. Clin Epigenetics 8:110

47. Spólnicka M, Piekarska RZ, Jaskuła E et al (2016) Donor age and C1orf132/MIR29B2C determine age-related methylation signature of blood after allogeneic hematopoietic stem cell transplantation. Clin Epigenetics 8:93. doi:10.1186/s13148-016-0257-7

48. Weidner CI, Ziegler P, Hahn M et al (2015) Epigenetic aging upon allogeneic transplantation: the hematopoietic niche does not affect age-associated DNA methylation. Leukemia 29:985-988. doi:10. 1038/leu.2014.323

49. Gratwohl A, Baldomero H, Aljurf M et al (2010) Hematopoietic stem cell transplantation: a global perspective. JAMA 303:16171624. doi:10.1001/jama.2010.491

50. Smith TJ, Hegedüs L (2016) Graves' disease. N Engl J Med 375: $1552-1565$

51. Garagnani P, Bacalini MG, Pirazzini C (2012) Methylation of ELOVL2 gene as a new epigenetic marker of age. Aging Cell 11: $1132-1134$

52. Horvath S (2013) DNA methylation age of human tissues and cell types. Genome Biol 14:R115

53. Kim J, Kim K, Kim H et al (2014) Characterization of age signatures of DNA methylation in normal and cancer tissues from multiple studies. BMC Genomics 15:997. doi:10.1186/1471-2164-15997 
54. Rojas R (1996) Neural networks: a systematicintroduction. Springer, Berlin

55. Tu JV (1996) Advantages and disadvantages of using artificial neural networks versus logistic regression for predicting medical outcomes. J Clin Epidemid 49:1225-1231

56. Basheer IA, Hajmeer M (2000) Artificial neural networks: fundamentals, computing, design, and application. J Microbiol Methods 43:3-31

57. Amato F, López A, Peña-Méndez EM et al (2013) Artificial neural networks in medical diagnosis. J Appl Biomed 11:47-58
58. Xu C, Qu H, Wang $\mathrm{G}$ et al (2015) A novel strategy for forensic age prediction by DNA methylation and support vector regression model. Sci Rep 5:17788. doi:10.1038/srep17788

59. Vidaki A, Ballard D, Aliferi A, Miller TH, Barron LP, Syndercombe Court D (2017) DNA methylation-based forensic age prediction using artificial neural networks and next generation sequencing. Forensic Sci Int Genet. doi:10.1016/j.fsigen.2017.02. 009 